\title{
A SIMPLE MODEL FOR BIOLOGICAL AGGREGATION WITH ASYMMETRIC SENSING*
}

\author{
PAUL A. MILEWSKI, XU YANG ${ }^{\dagger}$
}

\begin{abstract}
In this paper we introduce a simple continuum model for swarming of organisms in which there is a nonlocal aggregation term with an asymmetric sensing kernel countered by a nonlinear diffusion. The model can be thought of as capturing the dynamics of organisms that aggregate responding to visual cues and whose field of vision is predominantly in the direction of motion. The model has a variety of traveling solutions, including compact swarms where the speed of the swarm increases with the total number of organisms in the swarm (up to a largest swarm), traveling fronts and periodic waves. The dynamics of fully time-dependent solutions include complicated inelastic swarm interactions and the spontaneous breakup of small populations into compact swarms. We also comment on a kinetic formulation that leads to our equations and introduce a bi-directional model where the organisms are allowed to change directions.
\end{abstract}

Key words. swarming, chemotaxis, traveling waves

AMS subject classifications. 92D25, 92D50, 35Q80, 35F 25

\section{Introduction}

There exists a wide variety of models for the aggregation of organisms. These range from modeling the chemotaxis of bacteria, where the organisms sense variations in an ambient chemical attractant or repellent, to the social aggregation of higher organisms $[9,13]$. Here, we focus on an idealized simple continuum model for aggregation, and the traveling compact, periodic and front solutions resulting from the nonlocal sensing of nearby populations. We do not consider precise biological details on how individual behavior results in continuum aggregation-diffusion models (see $[11,4,5,10]$ ), since a goal is to analyze complex behavior arising from the simple equations. The model involves directional sensing, for example when the organism's response is to visual cues and the field of vision is predominantly in a particular direction, such as the organism's direction of motion. Such a nonlocal response from visual cues can be an important mechanism for the behavior of ungulate herds, schools of fish and insect swarms $[6,8,3,1]$. Directional sensing may also be applicable in modelling the more detailed dynamics of certain chemotactic processes where a subset of the population is moving and sensing only in a particular direction. For example, swimming bacteria have a memory of the spatial concentration of a chemoattractant behind them. First, we introduce the simple one-dimensional one-way model, in which the sensing is always in a fixed direction and organisms move preferentially in the direction they are sensing. The model allows for analytical analysis of the resulting solutions. We find that the model supports a one parameter family of compact traveling waves or "swarms" whose speed increases with the swarm size with a limiting, maximum-sized swarm. (Periodic arrays of swarms are also found.) In addition, the model has traveling front solutions, where a uniform large population level migrates into a region of lower population (or where singular swarms travel into a region of zero population), and periodic and solitary waves of organisms about a constant nonzero level. Using analysis and asymptotic arguments,

*Received: November 2, 2007; accepted (in revised version): March 24, 2008. Communicated by Jack Xin.

${ }^{\dagger}$ Department of Mathematics, University of Wisconsin, 480 Lincoln Dr., Madison, WI 53706 (milewski@math.wisc.edu, xyang@math.wisc.edu). 
we characterize these traveling solutions and discuss their stability. Numerical simulations of this simple one-way model are performed in a periodic domain, and show that sufficiently small initial data will spontaneously aggregate into swarms. Larger data will result in periodic solutions with $\rho>0$ everywhere. We also find that swarms of different size can coalesce in collisions that result in larger swarms together with some "radiation" (organisms left behind). We conclude by commenting on possible microscopic processes that would yield our equations in the drift-diffusion limit and introducing a bi-directional model in which a particular organism may change the direction in which it is sensing according to transition probabilities that depend on, for example, local population densities. In terms of biological relevance, there are two features of realistic organism aggregations that our swarm solutions reproduce: it has been observed that in fish schools, the speed of the fish increases with the density of the school [14], and that in ungulate herds, the density of the herd is largest at the "leading edge" [1]. On the other hand, it is clear that a simple one-dimensional model is expected to have only limited biological applications.

\section{Proposed model}

Consider a scalar conservation law model for the density of organisms $\rho(x, t) \geq 0$ of the form

$$
\rho_{t}+(\rho u(\rho))_{x}=0,
$$

where the speed of the organisms $u$ is modeled as having two contributions: a nonlocal attraction which will tend to nucleate swarms, together with a local repulsion that prevents the densities of organisms from growing too large. We shall choose in this paper, similarly to some models discussed in [8],

$$
u=K * \rho-\rho \rho_{x},
$$

with the kernel K defined by

$$
K=\left\{\begin{array}{l}
e^{x}, x \leq 0 \\
0, x>0
\end{array}\right.
$$

The convolution is defined as $K * \rho=\int_{-\infty}^{\infty} K(\xi) \rho(x-\xi) d \xi$, and, since $K$ has support for $\xi \leq 0$, the result of the convolution term is that a contribution to the speed of the organisms at a particular point is proportional to a weighted average of the organisms to the right of that point. Thus we are modeling right-sensing organisms, and an increasing population density to the right of an organism will tend to increase its locomotion speed. The nonlinear term in (2.2), proportional to the gradient of $\rho$, gives a contribution to the speed that is in the opposite direction of increasing $\rho$ and therefore leads to repulsion of nearby organisms.

Even though we chose a particular kernel for its simplicity in the analysis, it is expected (based on a few numerical experiments) that a wide class of asymmetric kernels could be used with similar results. We emphasize that the aggregation term is non-local and senses organisms at a distance $O(1)$ away from a particular point whereas the repulsion term is local. We have chosen all parameters to equal 1 in (2.2) without loss of generality: for $u=\alpha K(\beta x) * \rho-\gamma \rho \rho_{x}$ the parameters could be eliminated by a rescaling of $x, \rho$ and $t$. Furthermore, higher powers of $\rho$ in the nonlinear diffusive term are also amenable to the type of analysis we show here. 
In order to gain intuition for the dynamics and compare with a similar model with a symetric kernel [15], it is useful to consider the aggregation term in Fourier space. Then,

$$
\mathcal{F}\{K * \rho\}=\frac{1}{1-i k} \widehat{\rho}=\left(\frac{1}{1+k^{2}}+\frac{i k}{1+k^{2}}\right) \widehat{\rho}=\mathcal{F}\left\{\left(1+\partial_{x}\right) S * \rho\right\},
$$

where $\mathcal{F}(\rho)=\widehat{\rho}$ is the Fourier transform and $k$ the dual variable to $x . S=\frac{1}{2} \exp (-|x|)$ is the Poisson kernel whose Fourier transform is $\widehat{S}=\frac{1}{1+k^{2}}$. This kernel is the symmetric aggregation kernel used in [15].

In terms of $S$, our model could be written as

$$
\rho_{t}+[\rho(S * \rho)]_{x}+\left[\rho(S * \rho)_{x}-\rho^{2} \rho_{x}\right]_{x}=0,
$$

and, if the second term above is removed, one recovers the model in [15]. In their model, the dynamics is much simpler with all initial data tending to localized steady solutions. The additional term in our model clearly breaks the symmetry and allows for traveling solutions. It is useful to interpret physically the two nonlocal terms in (2.4). The term $\left[\rho(S * \rho)_{x}\right]_{x}$ is "anti-diffusive" and gives a contribution to the speed of the organisms that is proportional to the gradient of a locally symmetrically averaged population, resulting in aggregation. The term $[\rho(S * \rho)]_{x}$ is "advective" and gives a positive contribution to the speed proportional to the symmetrically averaged population. As we have mentioned before, in fish schools, the speed of the fish increases with the density of the school [14].

A further interpretation of the model is seen by writing the equation as a system for $\rho(x, t)$ and the local average population $w(x, t)=S * \rho$

$$
\begin{aligned}
\rho_{t}+[w \rho]_{x} & =\left[\rho^{2} \rho_{x}-\rho w_{x}\right]_{x}, \\
-w_{x x} & =(\rho-w) .
\end{aligned}
$$

Here it is clear that $w$ is a smoothed density which controls the advection and aggregation terms in the equation for $\rho$. At this point, we can make an analogy with chemotaxis. In certain swarming phenomena usually described by Keller-Segel type equations [7], bacteria swim up gradients in chemoattractants released by the bacteria themselves. In the limit where the diffusivity of the chemoattractant is fast compared to the bacterial "diffusivity", the Keller-Segel equations often take the form of the system (2.5) with a linear diffusive term and without the advective term for $\rho$.

\section{Traveling waves}

In this section we study the traveling wave solutions to our model, both from a direct analysis of the resulting equation under the traveling wave assumption and using phase plane methods. There are two types of solutions: singular solutions where the population density is zero somewhere in the domain and nonsingular solutions which are nowhere zero. There are many types of traveling waves: swarms with compact support, "rarefaction" fronts (corresponding to a decrease in population density with the passage of the front) and "invasion" fronts (corresponding to an increase in population density), periodic waves and noncompact solitary waves on a nonzero background. "Invasion" fronts are further divided into 2 types: those traveling into regions where $\rho=0$ ahead of the front and those traveling into regions of lower but nonzero uniform population. Periodic solutions also come in two types: those consisting of smooth solutions where everywhere $\rho>0$ and those consisting of 
a periodic array of compact swarms. Initially we restrict our discussion to solutions which propagate on the zero state such as a single swarm or a front propagating into the zero state. We then discuss fronts between two constant (nonzero) states, periodic solutions, and noncompact solitary solutions. Periodic solutions are in fact the ones we simulate in our time dependent computations, since these are performed on a periodic domain. In order to study the traveling wave solutions, we define

$$
v \equiv K * \rho=\int_{0}^{\infty} e^{-\xi} \rho(x+\xi) d \xi
$$

or equivalently,

$$
v_{x}=v-\rho, \quad v(0)=v_{0},
$$

and seek solutions $\rho=f(\theta)$ with $\theta=x-c t$. Then (2.1) becomes

$$
-c f^{\prime}+(f v)^{\prime}-\frac{1}{3}\left(f^{3}\right)^{\prime \prime}=0 .
$$

3.1. Singular solutions: compact swarms and singular fronts. Consider solutions where the population is nonzero only for $0<\theta<L$, that is, compact swarms of width $L$. Then, integrating once and assuming that the constant of integration is zero (which can be verified as consistent a posteriori), dividing by $f$, and integrating the result on an interval $\theta \in[0, L]$, we obtain

$$
-c L+\int_{0}^{L} v d \theta-\frac{1}{2}\left(f^{2}(L)-f^{2}(0)\right)=0 .
$$

Thus, with $f(0)=f(L)=0$, and defining the total mass of the swarm $M$ by

$$
M=\int_{0}^{L} f d \theta
$$

we then have

$$
c=\frac{1}{L} \int_{0}^{L} v d \theta=\frac{1}{L}\left(M-v_{0}\right) .
$$

We have used the fact that $v^{\prime}=v-f, v(L)=0$ and $v_{0}=v(0)$. In addition, from (3.3), carrying out similar steps without dividing by $f$, we can obtain:

$$
c=\frac{1}{M} \int_{0}^{L} f v d \theta=\frac{1}{M}\left(\int_{0}^{L} v^{2} d \theta+\frac{1}{2} v_{0}^{2}\right) .
$$

We first derive bounds on the speed c. Using the Hölder inequality $\left(\frac{1}{L} \int_{0}^{L} v d \theta\right)^{2} \leq$ $\frac{1}{L} \int_{0}^{L} v^{2} d \theta$ in (3.7), and eliminating $v_{0}$ using (3.6), we get

$$
\frac{M}{L+2} \leq c \leq \frac{M}{L} .
$$

We will sharpen the upper bound (see below) when looking at the solutions in the phase plane $(f, v)$. 

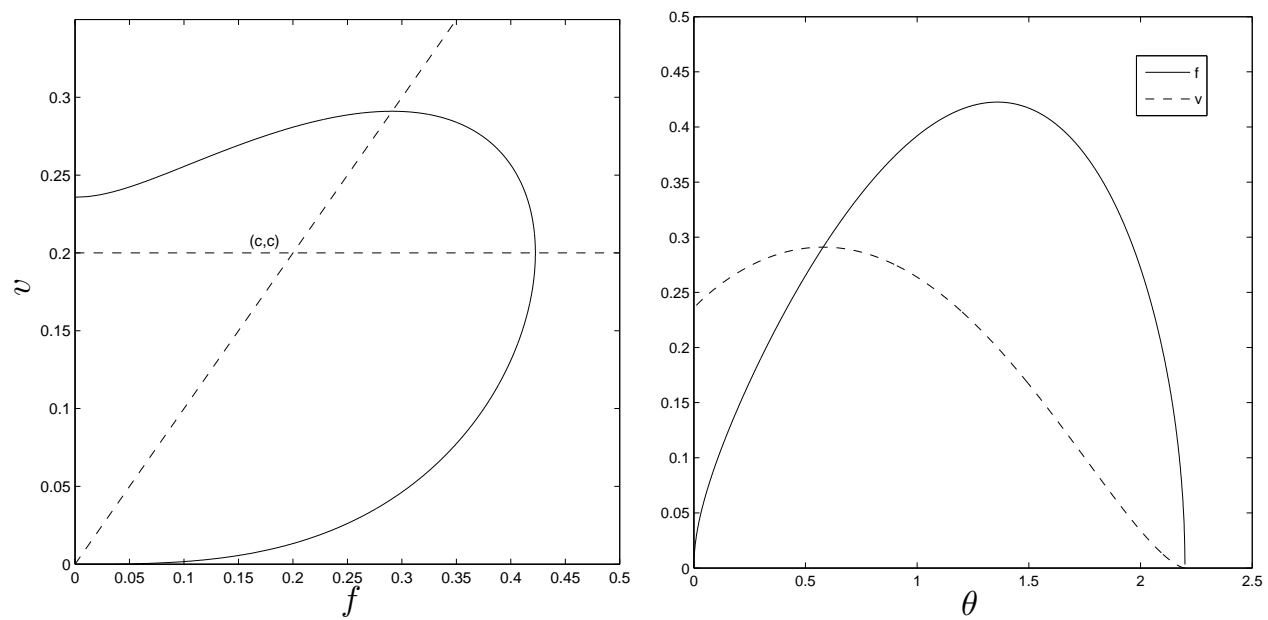

FIG. 3.1. Phase plane diagram (left) and traveling swarm solutions (right). In the phase plane, the dashed lines are the nullclines. Both $f$ and $v$ are shown. For this example $c=0.2$.

The phase plane for $f \geq 0, v \geq 0$ and for a fixed $c$ has solutions satisfying

$$
\begin{aligned}
& f^{\prime}=\frac{v-c}{f}, \\
& v^{\prime}=v-f .
\end{aligned}
$$

Typical solutions are shown in Figures 3.1 and 3.2. The flow has an unstable fixed point (spiral) at $(c, c)$ and is singular when $f=0$. This singularity allows for compact solutions of the form shown in the next section.

Not every solution curve in the phase plane corresponds to physical traveling solutions of (2.1). In addition to the fixed point, which corresponds to the constant solution (see Section 4 for the stability of this solution), only solutions limiting to $(0,0)$ as $\theta \rightarrow L$ are relevant. This is because if a solution has $f=0$ for $\theta \geq L$, then $v(L)$ must be zero by (3.1). As we shall see, this leaves two kinds of possible solutions, corresponding to swarms (compact solitary waves) and to fronts into the zero state (singular fronts). Swarm solutions are defined by the phase plane trajectory for $\theta \in$ $(0, L)$ that begin and terminate on the line $f=0$ connecting the points $\left(0, v_{0}\right)$ and $(0,0)$. For these solutions $f=0$ for $\theta \geq L$ and $\theta \leq 0, v=0$ for $\theta \geq L$, but $v \neq 0$ for $\theta \leq 0$ ( $v$ decays exponentially as $\theta \rightarrow-\infty$, by (3.1)). Figure 3.1 shows an example of such a solution. Front solutions are defined by trajectories in the phase plane for $\theta \in(-\infty, 0)$ that terminate at $(0,0)$ for $\theta=0$ but tend asymptotically to $(c, c)$ as $\theta \rightarrow-\infty$. For $\theta>0, f=v=0$. Figure 3.2 shows an example of such a solution. Of course, either the front or swarm solutions can be shifted arbitrarily in $x$.

For a given $c$ there is either a swarm or a front solution. Swarm solutions exist for $0<c \leq c^{*}$, whereas front solutions exist for $c>c^{*}$. Clearly, from the phase plane, for swarm solutions to exist, $v_{0} \geq c$ so that $f^{\prime}>0$. Physically, $v_{0}$ is the "attractive" part of the speed of the last organism due to the organisms ahead of it. Given that organisms will also feel some "repulsion" due to local density gradients, $v_{0}$ needs to be larger than $c$ if the last organism is to remain in the swarm and not fall behind. We shall see below that for vanishingly small swarms, $v_{0}=2 c$, and that $v_{0} / c$ decreases as $c$ increases. We then define $c^{*}$, the fastest swarm speed, as the value of $c$ with 

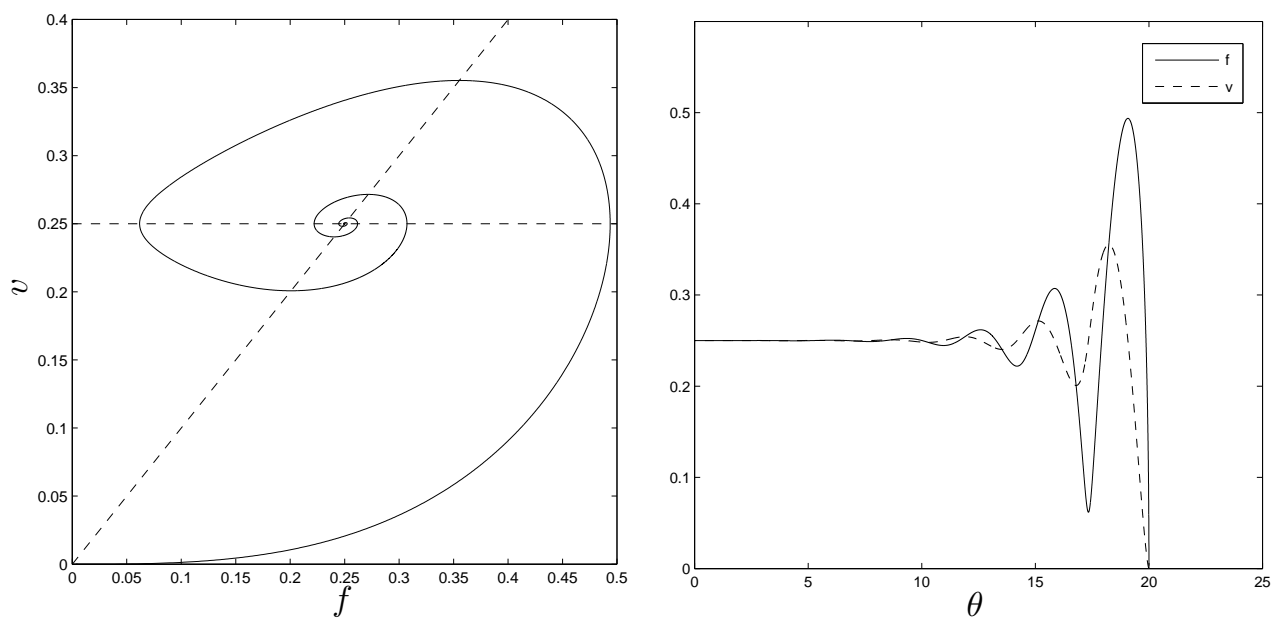

FIG. 3.2. Phase plane diagram (left) and traveling singular front solutions (right). In the phase plane, the dashed lines are the nullclines. Both $f$ and $v$ are shown. For this example $c=0.25$.

a swarm solution for which $v_{0} / c=1$. That is, the speed $c$ is such that the swarm trajectory in the phase plane is between $(0, c)$ at $\theta=0$ and $(0,0)$ at $\theta=L$. We can now sharpen the upper bound for the swarm speed, since $v_{0} \geq c$ implies, using (3.6), that $c \leq M /(L+1)$. Thus we have that

$$
\frac{M}{L+2} \leq c \leq \frac{M}{L+1} .
$$

To understand the transition from swarm solutions to front solutions at $c=c^{*}$, it is easier to think of reversing "time" in the phase plane, turning $(c, c)$ into a stable spiral. We can then ask what happens to solutions starting at $(0,0)$ (with the right asymptotics as derived in Section 3.2). For $c<c^{*}$ they terminate at $f=0$ above $v=c$, corresponding to the compact swarms. For $c>c^{*}$ they cross the nullcline $v=c$ for $f>0$ and spiral inwards to the fixed point $(c, c)$. These are traveling "invasive" fronts, corresponding to organisms traveling into an empty region. The fronts are not monotonic, having a decaying oscillatory component (see Figure 3.2). We find numerically that $c^{*} \approx 0.233$. In Figure 3.4 we show the comparison between the bounds (3.11), the computed values of $M / c$, and an approximation derived in Section 3.2 for $0<c<c^{*}$. Note that the bounds appear sharp.

From time dependent numerical computations it seems that individual solitary swarms are stable. Collisions between swarms may have complex behavior (see Section 4). Constant solutions, however, are only stable at large amplitude (see Section 4), and therefore one expects the front solutions to be unstable unless the constant state behind the front is sufficiently large.

We conclude this section with two observations. First, in time-dependent computations, we shall consider periodic solutions (with period $l$ ). Thus, we shall be computing periodic arrays of traveling swarms. These can be computed in the phase plane also: denoting by $\rho^{l}$ the function that is equal to the period-l function $\rho(x-c t)$ on $[0, l]$ and 0 otherwise (and assuming that $l>L$ where $L$ is the length of the swarm), 

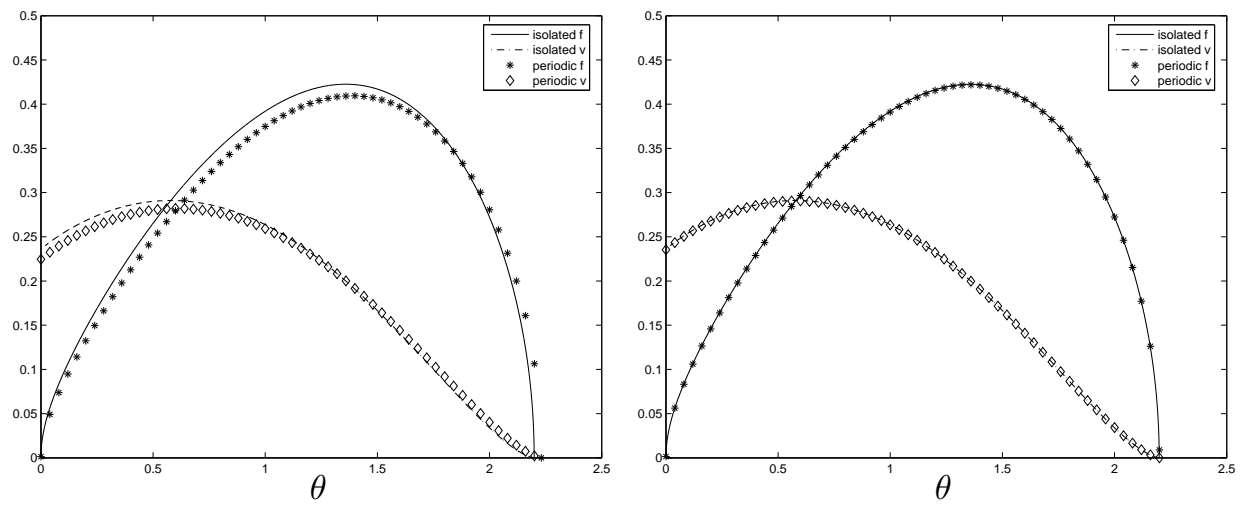

FIG. 3.3. Comparison of isolated (solid and dashed curves) and periodic ( $*$ and $\diamond$ curves) swarms for the same speed $c=0.2$. Both $f$ and $v$ are shown. Left: $l=5$, right: $l=8$.

the nonlocal term is modified:

$$
v=K * \rho=K * \rho^{l}+\frac{e^{-(l-\theta)}}{1-e^{-l}} v_{0}^{l} \equiv K * \rho^{l}+q\left(x ; l, v_{0}^{l}\right) .
$$

Thus the equations for the single copy of the periodic swarm are

$$
\begin{aligned}
& f^{l^{\prime}}=\frac{v^{l}+q\left(x ; l, v_{0}^{l}\right)-c}{f^{l}}, \\
& v^{l^{\prime}}=v^{l}-f^{l} .
\end{aligned}
$$

Given that the system now has $v_{0}^{l}$ as a parameter, it must now be solved by an iterative scheme (we used a simple shooting method). Figure 3.3 compares the infinite domain with the periodic domain solutions of the same speed $c$ in the infinite and in the periodic case. We see that the periodic solution has slightly smaller mass, which is intuitively reasonable given that it is sensing the swarms ahead of it. Also, the figure shows that as $l$ increases, the periodic swarm tends rapidly to the isolated one. A second observation is that, numerically, the phase plane pictures are computed by transforming the equations (3.10) using $g=f^{2} / 2$ and reversing time to give

$$
\begin{aligned}
& g^{\prime}=-v+c, \\
& v^{\prime}=-v+\sqrt{2 g} .
\end{aligned}
$$

The initial data is $(g, v)(0)=(0,0)$, and the trajectories either reach $\left(0, v_{0}\right)$ or spiral to $\left(c^{2} / 2, c\right)$. This transformation desingularizes $f=0$ and is more amenable to numerics.

3.2. Asymptotics for compact swarms. We present two kinds of asymptotic results in this section: first, the form of the swarms or fronts near their singular leading edge and the form of the swarm near the trailing edges, and then some results for small swarms.

Near the leading edge, at $x=L, v \approx 0$ in $(3.10)$ suggests that $f(x) \sim(L-x)^{1 / 2}$. Thus, substituting appropriate expansions in half-powers of $L-x$ yields, for $x<L$ :

$$
\begin{aligned}
f & \sim \sqrt{2 c}(L-x)^{1 / 2}-\frac{4}{15}(L-x)^{2}+o\left(|L-x|^{2}\right), \\
v & \sim \frac{2}{3} \sqrt{2 c}(L-x)^{3 / 2}-\frac{4}{15} \sqrt{2 c}(L-x)^{5 / 2}+o\left(|L-x|^{5 / 2}\right) .
\end{aligned}
$$


Near the trailing edge of the swarm, $v$ is nonzero, and the solutions are, locally for $x>0$,

$$
\begin{aligned}
& f \sim \sqrt{2\left(v_{0}-c\right)} x^{1 / 2}-\frac{v_{0}}{2 \sqrt{2\left(v_{0}-c\right)}} x^{3 / 2}+o\left(|x|^{3 / 2}\right), \\
& v \sim v_{0}+v_{0} x+o(|x|) .
\end{aligned}
$$

Estimates for the speed for small solutions $(M, L \ll 1)$ may be obtained by approximating the function $v(x)$ in terms of a cubic polynomial:

$$
v(x) \approx v_{0}+v_{0} x-v_{0} \frac{3+2 L}{L^{2}} x^{2}+v_{0} \frac{2+L}{L^{3}} x^{3},
$$

where the coefficients have been found from $v(0)=v^{\prime}(0)=v_{0}, v(L)=v^{\prime}(L)=0$, consistent with the approximations $(3.16,3.18)$ for $v$. Note that this approximate $v$ is positive but lacks the correct singularity at $x=L$. In other words, (3.17) is incompatible with (3.20) in the sense that in (3.17) $v^{\prime \prime}(L)$ is unbounded. Using (3.20) in (3.6) for small $L$, we obtain the results

$$
\begin{gathered}
v_{0} \sim 2 c\left(1-\frac{L}{6}\right), \\
\frac{M}{c} \sim 2+\frac{2}{3} L .
\end{gathered}
$$

Although this approximation is somewhat ad-hoc, it captures remarkably well the computed values for $M / c$ over the full range of amplitudes, as shown in Figure 3.4.

Since we have a one parameter family of solutions, we can find $L$ and $c$ as functions of $M$ alone by considering the expansions for $f$ in $(3.16,3.18)$, using (3.21), and calculating

$$
M \sim \frac{2}{3} \sqrt{c} L^{3 / 2}-\frac{19}{180} \sqrt{c} L^{5 / 2}+O\left(L^{3}\right) .
$$

From this relation, together with (3.22), we can obtain expressions for $c$ and $L$ as functions of $M$ :

$$
\begin{aligned}
L & \sim\left(\frac{9}{2}\right)^{1 / 3} M^{1 / 3}+\frac{13}{60}\left(\frac{9}{2}\right)^{2 / 3} M^{2 / 3}, \\
c & \sim \frac{M}{2}-\frac{1}{6}\left(\frac{9}{2}\right)^{1 / 3} M^{4 / 3} .
\end{aligned}
$$

The computed values of $c$ and $L$ together with these approximations are shown in Figure 3.5.

3.3. Nonsingular solutions: traveling fronts and waves. Other types of traveling solutions can be obtained if $\rho$ is never zero. Then we cannot set the constant resulting from the integration of (3.3) to zero. Thus,

$$
-c f+v f-\frac{1}{3}\left(f^{3}\right)^{\prime}=A,
$$




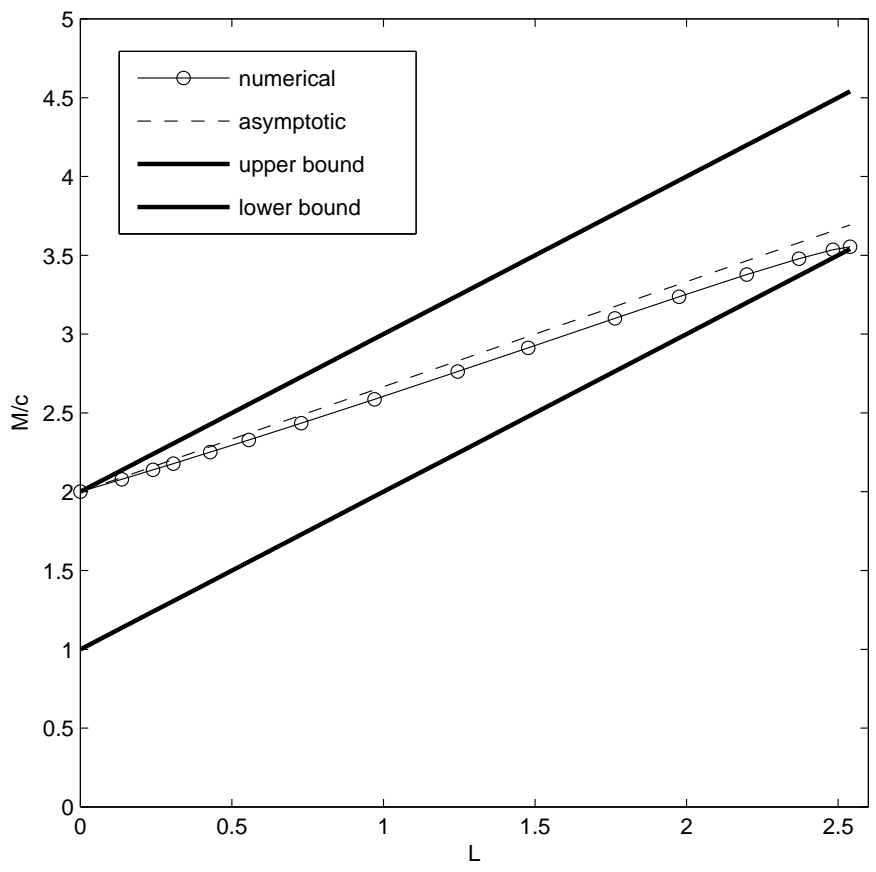

FIG. 3.4. Computed values of $M / c$ (solid line with circles) for traveling swarms. The upper and lower bounds (3.11) are the thick lines; the dashed line is the approximation for small L (3.22). The largest swarm has $c^{*} \approx 0.233, M^{*} \approx 0.826, L^{*} \approx 2.52$.
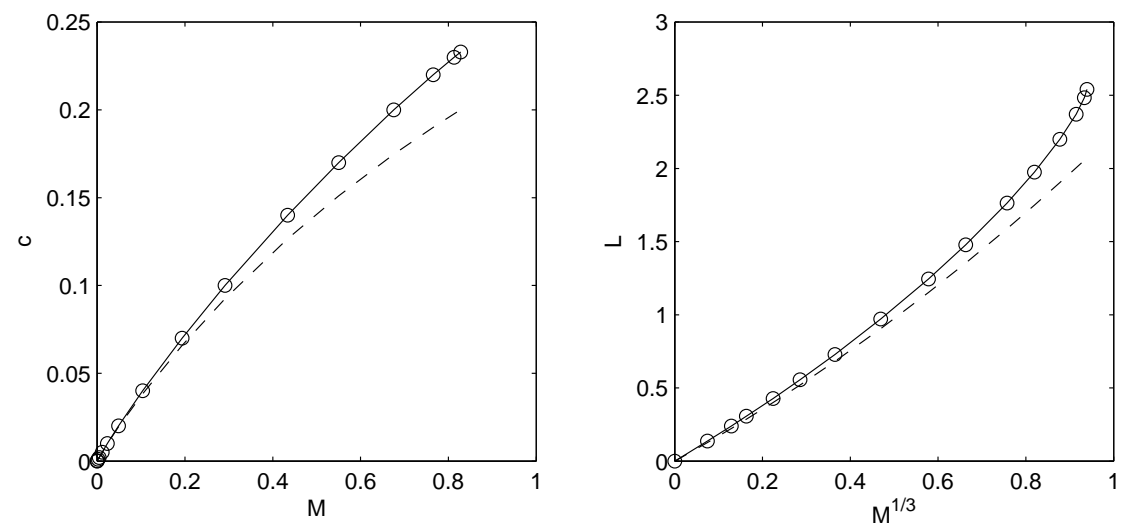

FIG. 3.5. Computed values of $c$ and $L$ as functions of $M$. The dashed lines are the approximations, valid for small $M$, in (3.24).

or, in the phase plane,

$$
\begin{aligned}
& f^{\prime}=\frac{v-c}{f}-\frac{A}{f^{2}}, \\
& v^{\prime}=v-f .
\end{aligned}
$$


We shall study the various solutions of this system in the $(c, A)$ parameter space.

The fixed points satisfy $v=f$ and therefore

$$
f^{2}-c f-A=0
$$

implying $f^{ \pm}=\frac{1}{2}\left(c \pm \sqrt{c^{2}+4 A}\right)$. For nonconstant traveling solutions, it is required that $A<0$. This is because for $A>0$ there is only one fixed point in the first quadrant, which rules out homoclinic or heteroclinic orbits. An argument ruling out periodic solutions in the case $A>0$ is given at the end of the section. Note that since $A<0$, $f>0,(3.28)$ implies that $c>0$.

The nullclines of this system are $v=f$ and $v=c+A / f$. The system undergoes a saddle-node bifurcation at $A=-c^{2} / 4$ where the two fixed points are created at $f=c / 2$. The Jacobian matrix $J$ of the linearization about fixed points is

$$
J=\left(\begin{array}{cc}
A / f^{3} & 1 / f \\
-1 & 1
\end{array}\right)
$$

Its eigenvalues satisfy the characteristic polynomial

$$
\lambda^{2}-T \lambda+D=0
$$

where $T=\left(1+\frac{A}{f^{3}}\right)$ and $D=\left(\frac{1}{f}+\frac{A}{f^{3}}\right)$ are the trace and determinant of $J$. Since

$$
D=(2 f-c) / f^{2}= \pm \frac{1}{\left(f^{ \pm}\right)^{2}} \sqrt{c^{2}+4 A}
$$

$f^{+}$is a node and $f^{-}$a saddle. The stability of $f^{+}$depends on the sign of $T . f^{+}$is neutrally stable when $T=0$, which implies that $f_{n}^{+}=(-A)^{1 / 3}$. Substituting this in the equation (3.28) for $f^{+}$, we obtain

$$
c=(-A)^{1 / 3}+(-A)^{2 / 3}
$$

as the neutral stability curve in the $(c, A)$ plane. This curve applies to $f^{+}$only for $0>A>-1$ (for $A<-1$ it is $f^{-}$that satisfies $T=0$ on this curve, which is irrelevant for the phase plane behavior). To decide stability, we calculate

$$
\frac{\partial T}{\partial A}=\frac{1}{f^{3}}-\frac{3 A}{f^{4}} \frac{\partial f}{\partial A} .
$$

From (3.28) we have that $\partial f / \partial A=(2 f-c)^{-1}$, therefore $\partial f^{+} / \partial A>0$, implying that for $A<0, \frac{\partial T}{\partial A}>0$. Thus the node $f^{+}$is stable for

$$
2(-A)^{1 / 2}<c<(-A)^{1 / 3}+(-A)^{2 / 3}, \quad 0>A>-1, \quad \text { Region I }
$$

and becomes unstable for $c>(-A)^{1 / 3}+(-A)^{2 / 3}$. The node $f^{+}$undergoes a Hopf bifurcation on the curve (3.32) when a stable limit cycle $C_{s}$ is born. Numerical evidence shows that this limit cycle grows in amplitude and disappears at a homoclinic bifurcation for $c=c_{h}(A)$ defined for $0>A>-1$. Thus, stable limit cycles exist for

$$
(-A)^{1 / 3}+(-A)^{2 / 3}<c<c_{h}(A), \quad 0>A>-1, \quad \text { Region II, }
$$

and when $c=c_{h}$ there is a homoclinic orbit from $f^{-}$circling $f^{+}$corresponding to a solitary wave of the system. For

$$
c>c_{h}(A), \quad 0>A>-1, \quad \text { or } \quad c>2(-A)^{1 / 2}, \quad A<-1 \quad \text { Region III, }
$$




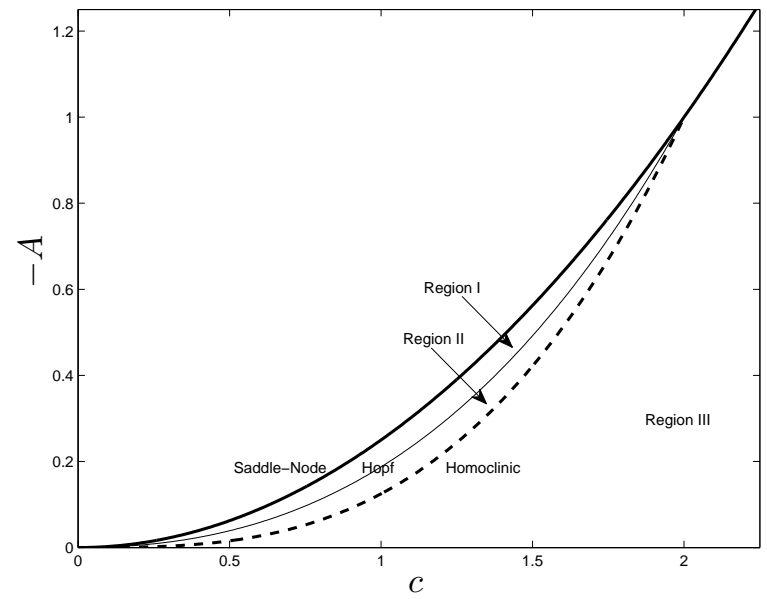

FIG. 3.6. Parameter space for $A<0$ solutions, indicating the bifurcations described in the text. The thick solid curve is $c=2(-A)^{1 / 2}$, above which there are no solutions. The thin solid curve is $c=(-A)^{1 / 3}+(-A)^{2 / 3}$, along which a limit cycle is born. Along the dashed curve $c=c_{h}(A)$, one has solitary wave solutions on a nonzero background. Note that the curves are only a sketch, since in reality the regions labeled are much narrower.

the fixed point $f^{+}$is unstable and there are no limit cycles in the phase plane. A sketch of these regions is given in Figure 3.6.

This overall bifurcation picture gives rise to many possible traveling waves. In the regime (3.34) there is a heteroclinic orbit with an oscillatory "rarefaction" front transition from $f^{-}$to $f^{+}$(see Figure 3.7). In the regime (3.35) there are three traveling wave solutions: orbits between $f^{-}$and $C_{s}, f^{+}$and $C_{s}$, and the periodic orbit $C_{s}$ (see Figure 3.8). When $c=c_{h}(A)$ there is the solitary homoclinic solution (see Figure 3.9) and one front-solitary transition solution connecting $f^{+}$to $C_{s}$. In the regime (3.36) there is a heteroclinic orbit with an oscillatory "invasive" front transition from $f^{+}$to $f^{-}$(see Figure 3.10).

Another interpretation of the front results arises from considering solutions of 3.25 tending to the different constants $f^{+}, f^{-}$at $\pm \infty$ (note that there, $v \rightarrow f$ ) yielding

$$
c=f^{+}+f^{-}>0, \quad A=-f^{+} f^{-}<0 .
$$

Thus the front speed for this problem is the same as the shock speed for the conservation law

$$
U_{t}+\left(U^{2}\right)_{x}=0,
$$

although the connection between the two states is much more complicated in the present case.

We finish this section by finding a lower bound on the speed of the periodic waves in terms of their period $L$ and mass (over a period) $M$ and showing that periodic solutions are not permitted for $A>0$ (front solutions are ruled out by the fact that there is only one fixed point in this case). 

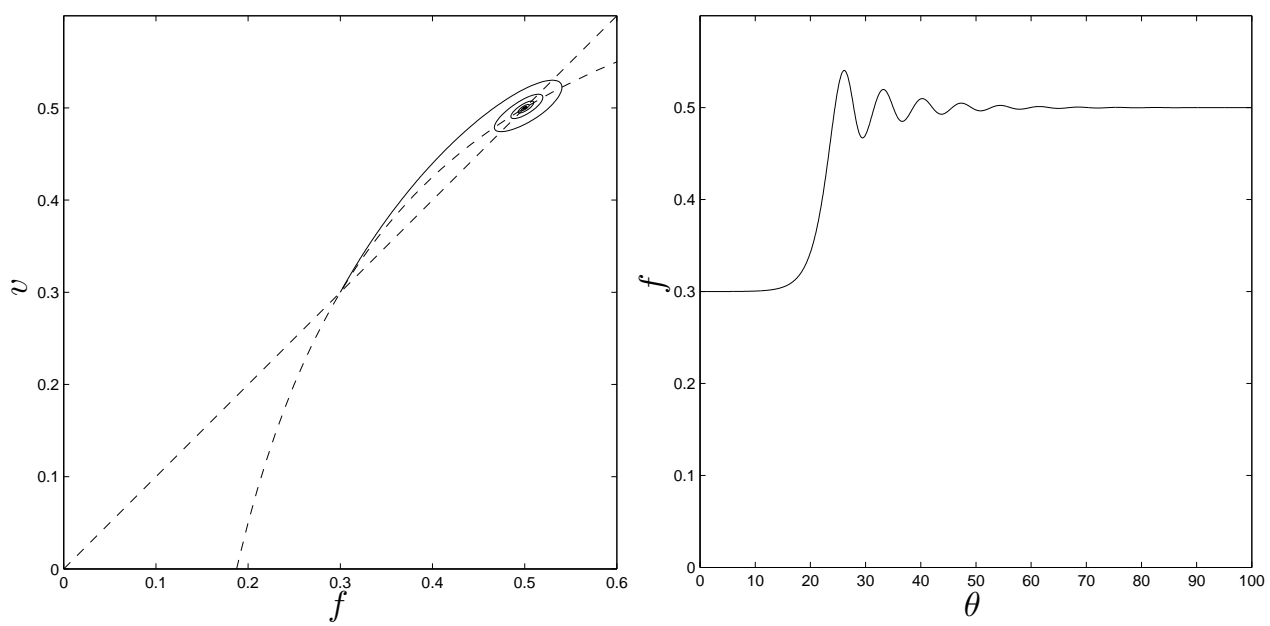

FIG. 3.7. Phase plane diagram (left) and traveling "rarefaction" front solutions (right) of the regime I in (3.34). In the phase plane, the dashed lines are the nullclines. For this example $c=0.8$, and $A=-0.15$.
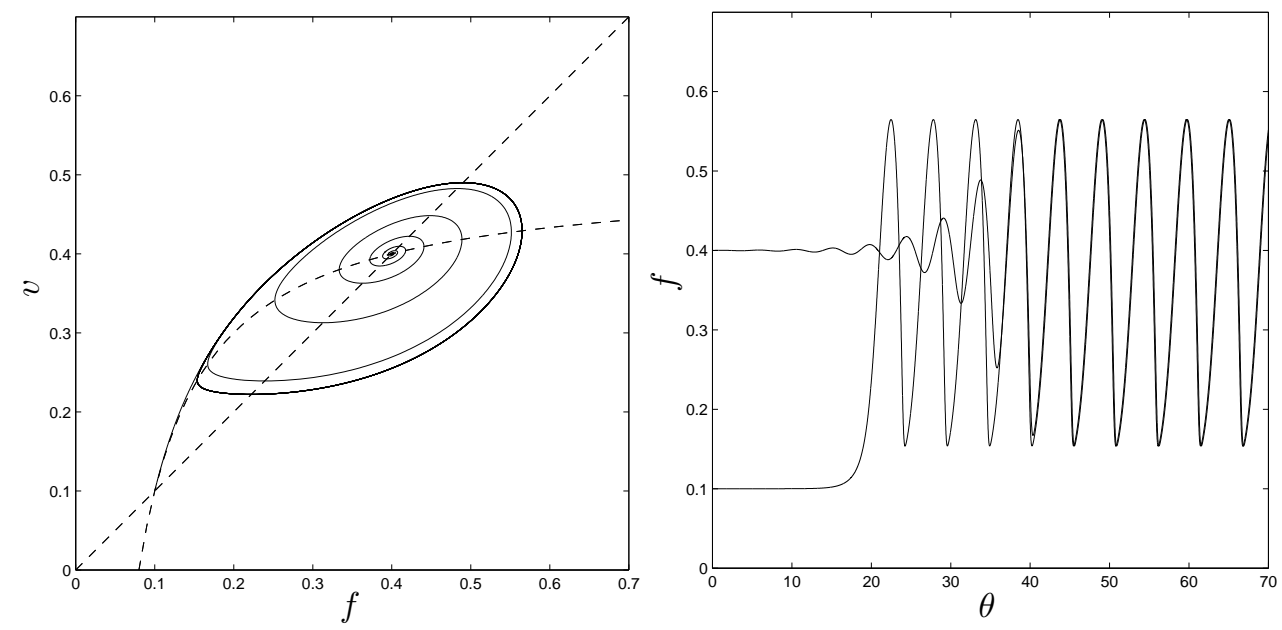

FIG. 3.8. Phase plane diagram (left) and constant-to-periodic solutions (right) of Region II in (3.35). In the phase plane, the dashed lines are the nullclines. Two of the three possible solutions are shown for this set of parameters. A uniform periodic solution is also possible. For this example $c=0.5$, and $A=-0.04$.

Consider, as before, (3.25) integrated over a period. Then

$$
-c M+\int_{0}^{L} v \rho d \theta=A L,
$$

and (3.25), divided by $\rho$ and integrated, gives

$$
-c L+M=A \int_{0}^{L} \frac{1}{\rho} d \theta .
$$



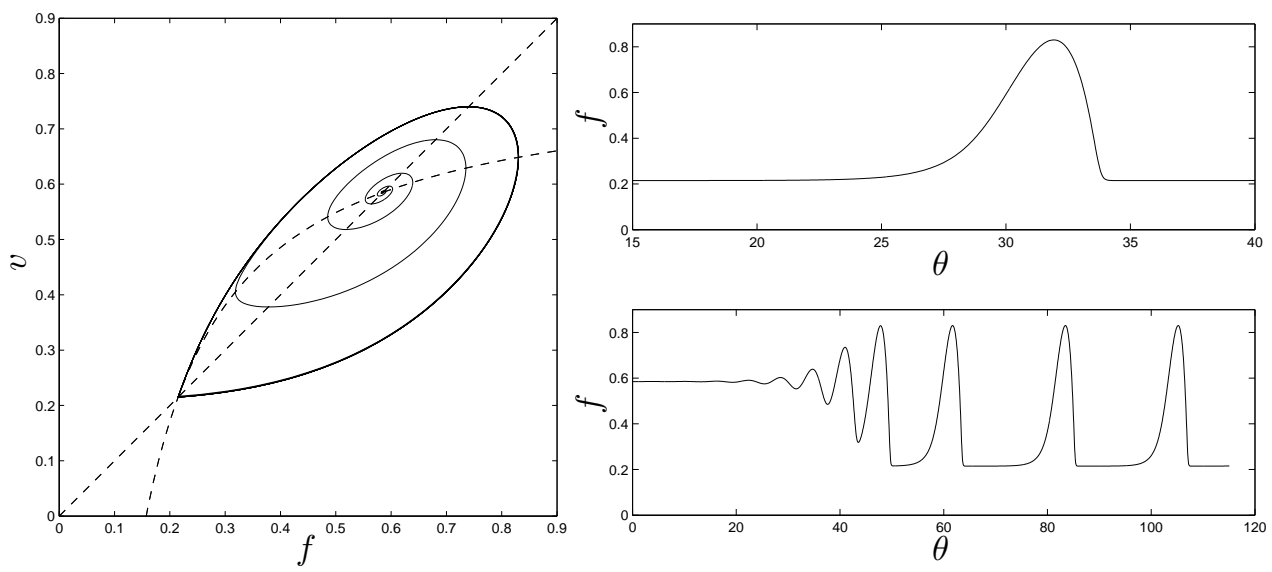

FIG. 3.9. Phase plane diagram (left) and solitary (right top) and front-solitary solutions (right bottom). In the phase plane, the dashed lines are the nullclines. For this example $c=0.8, A=$ -0.12575 .
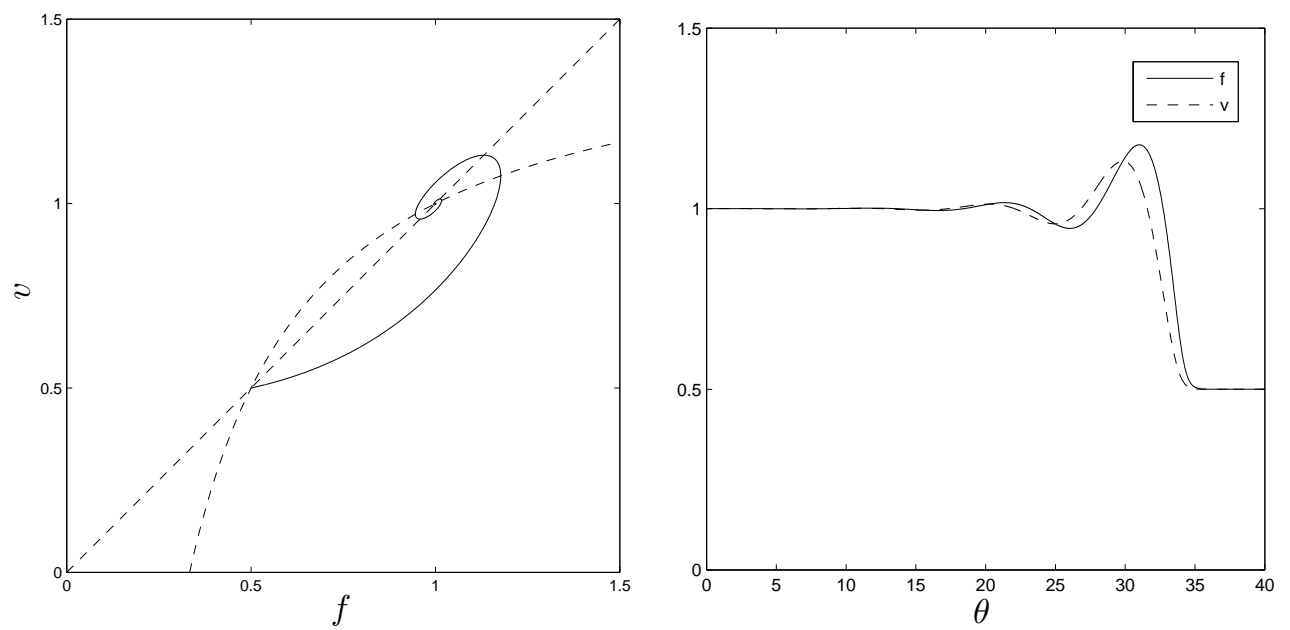

FIG. 3.10. Phase plane diagram (left) and traveling front solutions (right) of the regime III in (3.36). In the phase plane, the dashed lines are the nullclines. Both $f$ and $v$ are shown. For this example $c=1.5, A=-0.5$.

We have used that the integral of $v$ is equal to the integral of $\rho$. Now,

$$
\int_{0}^{L} v \rho d \theta=\int_{0}^{L} v^{2} d \theta \geq \frac{1}{L}\left(\int_{0}^{L} v d \theta\right)^{2}=\frac{M^{2}}{L} .
$$

Therefore

$$
A L \geq \frac{M^{2}}{L}-M c=\frac{M}{L}(M-L c) .
$$

Therefore, for the relevant case, $A<0$,

$$
c \geq M / L \text {. }
$$


Continuing, we can show that $A>0$ is impossible. Using the inequality (3.42) and (3.40) we get

$$
A L \geq \frac{M}{L} A \int_{0}^{L} \frac{1}{\rho} d \theta
$$

For $A>0$, from (3.44), we can conclude that $\int_{0}^{L} \frac{1}{\rho} d \theta \leq \frac{L^{2}}{M}$. However,

$$
L^{2}=\left(\int_{0}^{L} \frac{1}{\sqrt{\rho}} \sqrt{\rho} d \theta\right)^{2} \leq \int_{0}^{L} \frac{1}{\rho} d \theta \int_{0}^{L} \rho d \theta=M \int_{0}^{L} \frac{1}{\rho} d \theta .
$$

This contradicts the previous inequality unless $\rho$ is constant, and therefore we can conclude that no periodic solutions are possible for $A>0$.

\section{Time dependent solutions}

The time dependent equation, as in the traveling wave case, has a very rich behavior. Here we demonstrate a range of these behaviors and interpret them using the traveling wave solutions we have found and a linear stability analysis. The timedependent computations are carried out on a periodic domain with the nonlocal term computed in Fourier space and explicitly in time, and the local, nonlinear term treated conservatively and implicitly in time. The singular solutions require some care to ensure accurate computations.

First, consider a linearization about the constant solution of the form $\rho=\rho_{0}+$ $\delta e^{\sigma t} e^{i k x}$; we obtain that the perturbation has a growth rate

$$
\frac{\sigma}{\rho_{0}}=\frac{k^{2}}{1+k^{2}}\left(1-\rho_{0}\left(1+k^{2}\right)\right)-i k \frac{2+k^{2}}{1+k^{2}} .
$$

This means that there is an instability within a band of wavenumbers $k \in\left(0, k^{*}\right)$ for $\rho_{0}<1$, and that for $\rho_{0}>1$ there is no instability. The shortest unstable wave has $k^{*}=\sqrt{\left(1-\rho_{0}\right) / \rho_{0}}$. The maximum growth rate occurs at $k_{\max }=\sqrt{1 / \rho_{0}}-1$ and there, $\operatorname{Re} \sigma_{\max }=\rho_{0}\left(1-\sqrt{\rho_{0}}\right)^{2}$.

In Figure 4.1 we show the evolution from a constant state with a small perturbation. The solution eventually aggregates into a compact swarm. This behavior of aggregating into one or more compact swarms is universal for sufficiently small initial data. If the initial data is larger and yet still unstable, the solution tends to periodic traveling waves. Given a domain of length $l$, if $m$ is the mass of the initial data over a period and $M(L)$ is the mass of infinite domain swarms of length $L$, then if

$$
m>\min \left(M^{*}, M(l)\right)
$$

we can rule out that the solution will tend to a single compact swarm. In Figure 4.2 we show a situation where the initial data is larger than in Figure 4.1 and the solution tends to a smooth periodic traveling wave. From our numerical experiments, the ultimate state of the system depends on both the initial mass and on the period $l$ (especially for $l$ small), leading always to either trains of compact swarms or to periodic waves.

The interaction of compact swarms on the infinite line is also interesting. Numerical experiments show that the collision of two swarms tends to form a single larger swarm and leaves behind some "radiation" that reforms into a smaller swarm. An example of such collisions is shown in Figure 4.3. 

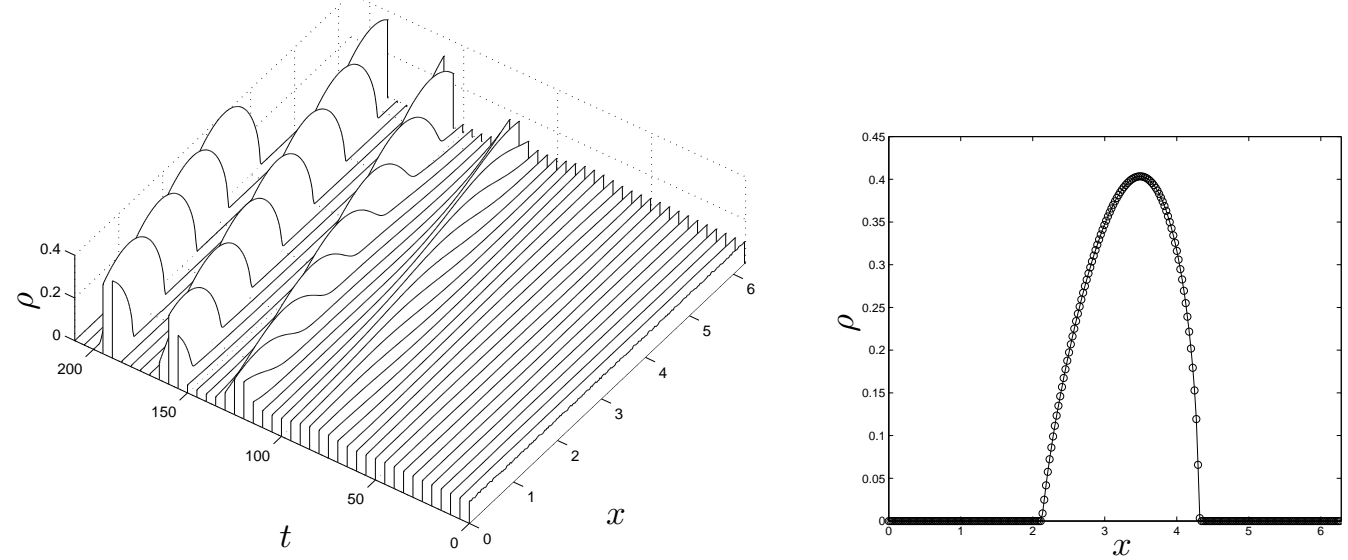

FIG. 4.1. Linear instability of the constant state leading to the formation of a traveling compact swarm. Initially $\rho=0.1$ with a small perturbation. Note that $m=0.63<M^{*}$
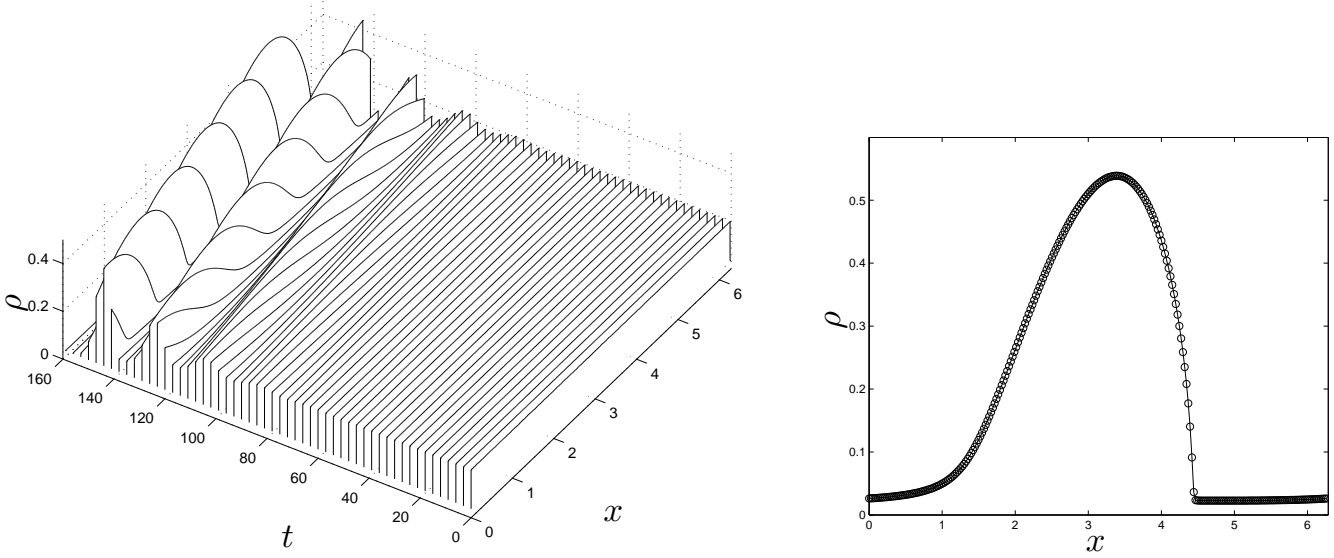

FIG. 4.2. Linear instability of the constant state leading to the formation of a traveling periodic wave. Initially $\rho=0.2$ with a small perturbation. Note that $m=1.26>M^{*}$. Note the similarity of the final state with the solitary wave solution of Figure 3.9.

Given that constant states with $0<\rho_{0}<1$ are unstable, we can now discuss the stability of the various front solutions found in Section 3.3. From (3.28), $f=1$ when $c=1-A$. In Figure 4.4 we show how this line defines regions where both, one or no fixed points are linearly stable. In the region where $f^{ \pm}>1$, which lies in Region III of Figure 3.6, we expect that front solutions of the type shown in Figure 3.10, with both states larger than 1 , are stable. The region $f^{ \pm}<1$, we can show, includes Region I in Figure 3.6 and, numerically, it appears that it also includes Region II (i.e., $c_{h}(A)<1-A$ ). Thus we expect solutions of the type shown in Figures 3.7, 3.8 and 3.9 to be unstable. However, periodic traveling solutions appear to be stable and attracting according to numerical calculations. Although these arguments also lead to the conclusion that solitary waves on the real line are unstable, periodic solutions of long period and large amplitude, which are very close to the solitary wave solution, 

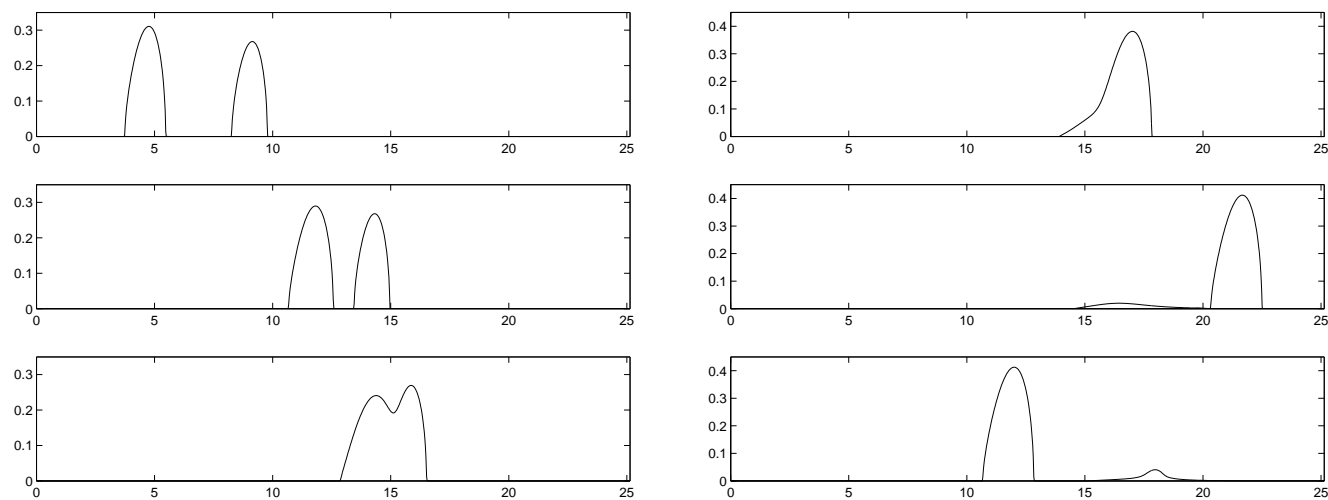

Fig. 4.3. Collisions of compact swarms in the $x-\rho$ plane. Snapshots of the collision of 2 swarms of different mass. The total mass is 0.705 , less than $M^{*}$. On the left, $t=20,70,85$. On the right, $t=95,120,200$. Note that: (i) As the faster swarm approaches the smaller one it deforms before reaching it due to the nonlocal term. (ii) The collision creates a larger swarm (the scale on the right is different) and leaves behind some "radiation". (iii) The radiation is aggregating (and due to periodicity of the domain the larger swarm has wrapped around).

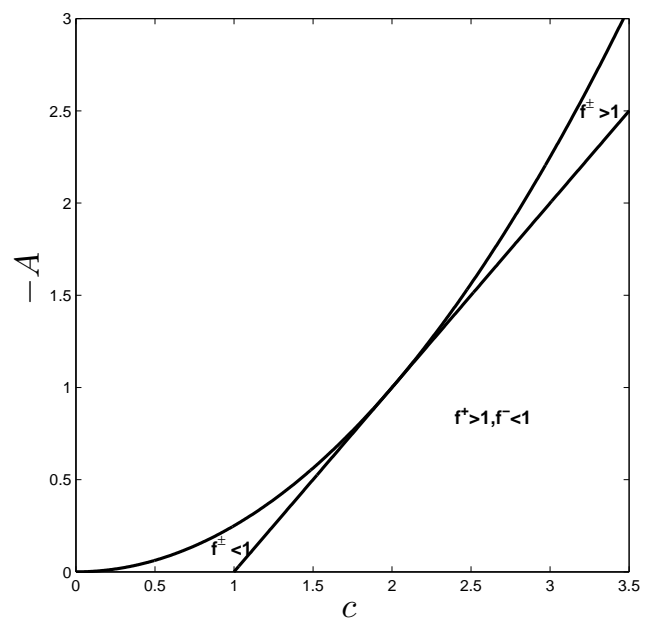

FIG. 4.4. Parameter space for stability of constant states.

seem to be stable (see, for example Figure 4.2). This phenomenon appears to be due to the fact that the periodic "soliton" sweeps through the domain quickly, destroying any growing instability which travels more slowly and which, since the background density is low, has small growth rate.

When $f^{+}>1, f^{-}<1$, which is also in Region III of Figure 3.6, the solutions of the type shown in Figure 3.10 are also possible. Ahead of the front, $f^{-}<1$, and therefore we can expect instability if the perturbations extend to $+\infty$. However, if perturbations are restricted to be localized near the front, we conjecture that the front is stable. For singular fronts (see Section 3.1), if the state behind the front has $\rho<1$, we expect it to be unstable. Examples of the propagation of singular fronts and their (in)stability are shown in Figure 4.5. 

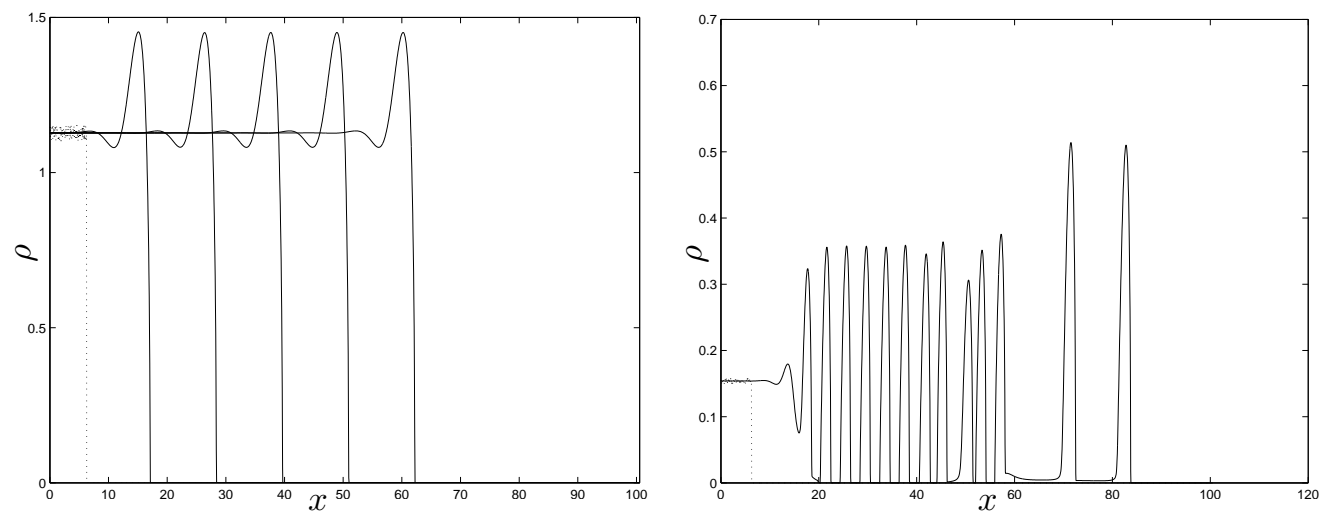

FIG. 4.5. Two examples of singular front propagation. The initial data (dotted curve) is a nonzero constant state for $x<2 \pi$ and zero for $x>2 \pi$ with a random perturbation for $0<x<2 \pi$. Left: Solutions at various times when the state behind the front has $\rho>1$ and the dynamics leads to a stable front. The solution is shown at $t=0,10,20,30,40,50$. Right: Solutions when the state behind the front is unstable $(\rho<1)$ and the front breaks up into a combination compact swarms and nonsingular oscillations. The solution is shown at $t=0$ and $t=400$.

\section{Kinetic and bi-directional models}

One may ask whether the model $(2.1,2.2)$ we present represents a certain scaling limit of a microscopic particle model $[12,2]$. Consider the transport equations for the probability $p^{ \pm}(x, t)$ of finding organisms moving at constant velocities $\pm s$ on the real line and reversing directions according to a Poisson process

$$
\begin{aligned}
& \frac{\partial p^{+}}{\partial t}+s \frac{\partial p^{+}}{\partial x}=-\lambda^{+} p^{+}+\lambda^{-} p^{-}, \\
& \frac{\partial p^{-}}{\partial t}-s \frac{\partial p^{-}}{\partial x}=-\lambda^{-} p^{-}+\lambda^{+} p^{+} .
\end{aligned}
$$

Defining the average reversal probability $\lambda=\left(\lambda^{+}+\lambda^{-}\right) / 2$ and the bias $\delta=\lambda^{+}-\lambda^{-}$, the total density $p=p^{+}+p^{-}$and the flux of organisms $q=s p^{+}-s p^{-}$satisfy

$$
\begin{gathered}
\frac{\partial p}{\partial t}+\frac{\partial q}{\partial x}=0 \\
\frac{\partial q}{\partial t}+s^{2} \frac{\partial p}{\partial x}=-s \delta p-2 \lambda q .
\end{gathered}
$$

In the drift-diffusion limit $s, \lambda, \delta \rightarrow \infty$ with $\lambda=s^{2} /\left(2 p^{2}\right)$ and $\delta=-s(K * p) / p^{2}$, we formally obtain

$$
q=p K * p-p^{2} p_{x} .
$$

Assigning $p=\rho$, this is the flux law we assumed in (2.2) and together with the conservation law for the total density yields $(2.1,2.2)$. Although this model is "bidirectional" at the microscopic level, the bias due to sensing is strictly unidirectional. This should be contrasted with a different macroscopic bidirectional model presented below, where organisms are allowed to sense in both directions.

In the models we have presented so far, the sensing is always in the same direction. In certain applications a more realistic model should include the ability of 


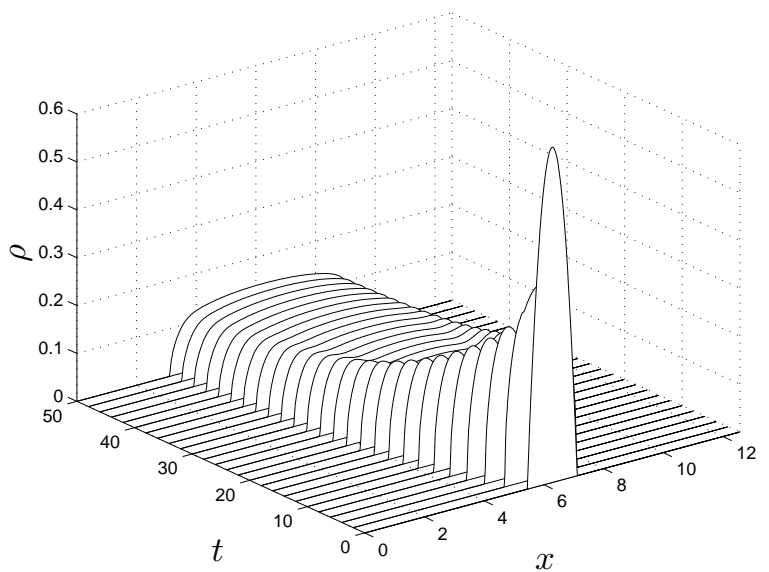

FIG. 5.1. Spreading of localized initial data in a bi-directional model (5.1) with a Poisson process for turning. Here, $\alpha=2$ and $\beta=0$.

organisms to reverse their direction of sensing. One possibility is to consider separate "populations" of right- and left-sensing organisms. Using the densities $\rho^{+}$and $\rho^{-}$, respectively, introducing the sensing kernels $K^{+}(x)=K(x), K^{-}=-K(-x)$ and modeling a macoscopic turning process, we can write, for example,

$$
\begin{aligned}
& \rho_{t}^{+}+\left[\rho^{+}\left(K^{+} * \rho-\rho \rho_{x}\right)\right]_{x}=-\alpha\left(\rho^{+}-\rho^{-}\right)+\beta \rho^{+} \rho^{-}\left(\rho^{+}-\rho^{-}\right), \\
& \rho_{t}^{-}+\left[\rho^{-}\left(K^{-} * \rho-\rho \rho_{x}\right)\right]_{x}=-\alpha\left(\rho^{-}-\rho^{+}\right)+\beta \rho^{+} \rho^{-}\left(\rho^{-}-\rho^{+}\right) .
\end{aligned}
$$

The term proportional to $\alpha$ corresponds to a Poisson process for organisms turning around and the $\beta$ term corresponds to organisms turning when there is a large density moving in the opposite direction. Note that in the flux, we have chosen the speed to depend on the full population, and note that the system preserves the total number of organisms. For $\alpha=0$, if one of the $\rho$ is initially zero, then it remains zero and the dynamics reduces to the one-way model we have discussed. In Figure 5.1 we show an example of a calculation with $\beta=0$ where a localized mixed population spreads.

In order to recover the bidirectional model (5.1) from a microscopic process similar to the one described above, one would introduce four probability density functions (each population with a given sensing direction would have left- and right-moving organisms). One assumes that there would have to be a scale separation between the microscopic Poisson processes and the macroscopic ones that reverse sensing.

\section{Conclusion}

We have introduced a simple continuum swarming model with directional sensing which leads to the self-aggregation of organisms into compact traveling swarms, when local densities are sufficiently small. Swarm speed increases with the swarm size up to a largest possible swarm. At larger organism densities one observes the breakup of a large swarm into smaller ones or periodic, traveling waves depending on the boundary conditions. Between regions of different organism densities there are front solutions. The model is simple enough that one can make substantial progress at understanding the solutions analytically. We summarize the parameter ranges for the various cases in Table 6.1. 


\begin{tabular}{|l|l|l|}
\hline \multicolumn{1}{|c|}{ Traveling Solutions } & \multicolumn{1}{|c|}{ Parameters } & \multicolumn{1}{c|}{ Properties } \\
\hline singular compact swarms & $A=0, \quad 0 \leq c \leq c^{*}$ & $\begin{array}{l}\text { Stable and have a maxi- } \\
\text { mum size when } c=c^{*} .\end{array}$ \\
\hline singular invasion fronts & $A=0, \quad c>c^{*}$ & $\begin{array}{l}\text { Stable only if the constant } \\
\text { state behind is sufficiently } \\
\text { large. }\end{array}$ \\
\hline $\begin{array}{l}\text { nonsingular traveling swarms } \\
\text { periodic or solitary) }\end{array}$ & condition $(3.34)$ & $\begin{array}{l}\text { Stable. Limit cycle is } \\
\text { born when } c=(-A)^{1 / 3}+ \\
(-A)^{-2 / 3} \text { Solitary wave } \\
\text { appears when } c=c_{h}(A) .\end{array}$ \\
\hline nonsingular rarefaction fronts & condition $(3.35)$ & $\begin{array}{l}\text { Stable only if the constant } \\
\text { state behind is sufficiently } \\
\text { large. }\end{array}$ \\
\hline nonsingular invasion fronts & condition (3.36) & $\begin{array}{l}\text { Stable only if the constant } \\
\text { state behind is sufficiently } \\
\text { large. }\end{array}$ \\
\hline
\end{tabular}

TABLE 6.1. A summary list for traveling solutions, their parameters and properties.

We also comment on a microscopic model that yields our equations in the appropriate limits and on a possible extension to a bidirectional model.

Acknowledgements. The work of P.A. Milewski and of X. Yang were partially supported by grants from the Division of Mathematical Sciences at the National Science Foundation (DMS-0608720 and DMS-0604635) and by NIGMS.

\section{REFERENCES}

[1] I.D. Couzin and J. Krause, Self-Organization and Collective Behavior in Vertebrates, Advances in the Study of Behavior, Academic Press, 32, 2003.

[2] F. Chalub, P. Markowich, B. Perthame and C. Schmeiser, Kinetic Models For Chemotaxis and Their Drift-Diffusion Limits, Nonlinear Differential Equation Models, Springer, 2004.

[3] L. Edelstein-Keshet, J. Watmough and D. Grunbaum, Do traveling band solutions describe cohesive swarms? An investigation for migratory locusts, J. Math. Biol., 36, 515-549, 1997.

[4] D. Grunbaum, Translating stochastic density-dependent individual behavior to a continuum model of animal swarming, J. Math. Biol., 33, 139-161, 1994.

[5] D. Grünbaum and A. Okubo, Modelling social animal aggregations, S. Levin (Ed.), Frontiers in Mathematical Biology, Lecture Notes in Biomathematics, Springer-Verlag, Berlin, 100, 296-325, 1994.

[6] K. Kawasaki, Diffusion and the formation of spatial distributions, Math. Sci., 16(183), 47-52, 1978.

[7] E.F. Keller and L.A. Segel, Traveling bands of chemotactic bacteria: a theoretical analysis, J. Theor. Biol., 30, 235-248, 1971.

[8] A. Mogilner and L. Edelstein-Keshet, A non-local model for a swarm, J. Math. Biol., 38, 534-570, 1999.

[9] J. Murray, Mathematical Biology, II, Springer, 1993.

[10] A. Okubo and S. Levin, Diffusion and Ecological Problems, 2nd edition, Inter. Appl. Math., Math. Biol., Springer, New York, 14, 2001.

[11] H.G. Othmer, S.R. Dunbar and W. Alt, Models of dispersal in biological systems, J. Math. Biol., 26, 263-298, 1988. 
[12] H.G. Othmer and T. Hillen, The diffusion limit of transport equations II: Chemotaxis equations, SIAM J. Appl. Math., 62, 1222-1250, 2002.

[13] J. Parrish and W. Hamner, Animal Groups in Three Dimensions, Cambridge University Press, Cambridge, UK, 1997.

[14] T.J. Pitcher and B.L. Partridge, Fish school density and volume, Marine Biology, 54(4), 383394, 1979.

[15] C.M. Topaz, A.L. Bertozzi and M.A. Lewis, A nonlocal continuum model for biological aggregation, Bulletin of Mathematical Biology, 68(7), 1601-1623, 2006. 